\title{
Energy efficient virtual machine provisioning in cloud data centers
}

\begin{abstract}
A cloud data center consumes a great amount of electrical energy, resulting in a high level of carbon footprint. The reduction of electrical power consumption cuts down operational costs, and also improves system reliability for cloud based data centers. Data center power consumption is a critical issue that could be solved by consolidating servers' workload and turning off idle physical servers. We propose a heuristic based resource allocation policy for cloud based data centers in order to reduce operational costs. Our proposed technique shows that efficient resource allocation greatly reduces energy consumption, which leads to minimize operational costs. One of the more significant findings to emerge from this study is that, based on CPU utilization and live migration, proposed algorithm helps to minimize energy costs. The present study provides additional evidence with respect to environmental issues.
\end{abstract}

Keyword: Data center; Migration of virtual machines; Power consumption; Power saving; Virtualization 